\title{
Rise and fall of thyroid radiologic density during the time course of amiodarone-induced thyrotoxicosis
}

\author{
Laure Felix ${ }^{1}$, Peggy Jacon², Maxime Lugosi ${ }^{3}$, Justine Cristante ${ }^{5}$, Julie Roux ${ }^{4}$ and Olivier Chabre $^{5}$ \\ 1 Univ. Grenoble Alpes, Department of Radiology, Centre Hospitalier Universitaire Grenoble Alpes, Grenoble, France \\ ¿Univ. Grenoble Alpes, Department of Cardiology, Centre Hospitalier Universitaire Grenoble Alpes, Grenoble, France \\ 3Univ. Grenoble Alpes, Department of Internal Medicine, Centre Hospitalier Universitaire Grenoble Alpes, Grenoble, France \\ ${ }^{4}$ Univ. Grenoble Alpes, Department of Nuclear Medicine, Centre Hospitalier Universitaire Grenoble Alpes, Grenoble, France \\ 5Univ. Grenoble Alpes, Department of Endocrinology, Centre Hospitalier Universitaire Grenoble Alpes, Grenoble, France
}

Correspondence should be addressed to O Chabre: OlivierChabre@chu-grenoble.fr

\begin{abstract}
Background and Objective: It has been reported recently in a cross sectional study that patients with amiodarone induced thyrotoxicosis (AIT) showed a 'white' thyroid on unenhanced computed tomography, due to intrathyroid iodine accumulation. However, the link between increase in thyroid radiologic density and amiodarone induced thyrotoxicosis remains unknown. We sought to analyze this link.

Methods: We present the case of a 34-year-old patient with severe sarcoidosis-related hypertrophic cardiomyopathy who was followed with successive unenhanced CT scans integrated with FDG PET scans. After the first CT scans the patient, who initially had a normal thyroid function, was exposed to amiodarone during 23 months and developed AIT, very likely by thyroiditis (AIT type 2). There were no thyroid antibodies, no evidence of thyroid sarcoidosis on FDG PET scan, while thyroid sonogram showed a homogenous $22 \mathrm{ml}$ moderate goiter with normal echogenicity and no nodules.

Results: Analysis of the successive enhanced CT scans revealed that after initiation of amiodarone treatment, thyroid radiologic density steadily increased before detection of AIT, peaked after cessation of amiodarone and initiation of thyrotoxicosis treatment, before returning to normal as thyrotoxicosis receded. Thyroid volume also showed a moderate increase, peaking at the detection of thyrotoxicosis, before returning to normal. Conclusion: This case suggests that AIT is preceded by a very high intrathyroid iodine accumulation before the 'burst' of thyroiditis occurs and that measurements of thyroid gland radiological density might predict the development and remission of AIT.
\end{abstract}

We present here the case of a 34-year-old patient with severe sarcoidosis-related cardiomyopathy who developed amiodarone-induced thyrotoxicosis (AIT). Evolution of sarcoidosis was closely monitored by fluorodeoxyglucosepositron emission tomography (FDG-PET) with integrated unenhanced CT scan. Although there was no thyroid signal on FDG PET, we noticed that in the unenhanced CT scan the thyroid had become 'white', suggesting that intrathyroid accumulation of iodine brought by amiodarone acted like an endogenous contrast medium, a phenomenon recently reported (1).

The originality in our observation is that we could go back to the unenhanced CT scans performed before exposure to amiodarone and we could follow the evolution of thyroid radiologic density on the subsequent CT scans, until remission of thyrotoxicosis. This provided 


\section{| European Thyroid}
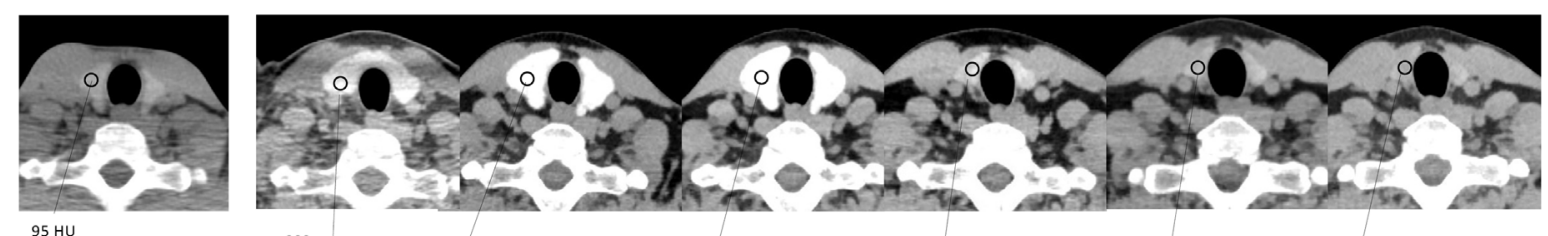

before first $3 \mathrm{mths}$ amiodarone treatment

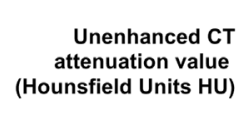

$200[1 / 20$

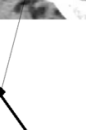

.

180

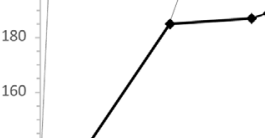

(Hounsfield Units HU)
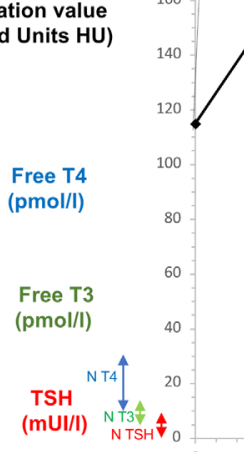

西

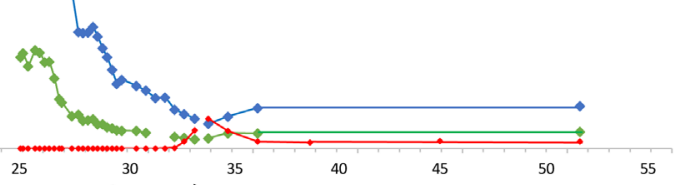

Months after initiation

$10 \quad 15 \quad 20$

40

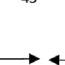

\begin{tabular}{c}
\hline Amiodarone $200 \mathrm{mg} / \mathrm{d}$ \\
\hline
\end{tabular}

$\underset{\text { Prednisone } 0.5 \text { to } 0.2 \mathrm{mg} / \mathrm{kg} / \mathrm{d}}{\text { Propylthiouracil } 800 \text { to } 50 \mathrm{mg} / \mathrm{d}} \longrightarrow$

Infliximab $5 \mathrm{mg} / \mathrm{kg} / \mathrm{m}$ ths; methotrexate $20 \mathrm{mg} /$ weeks

\section{Figure 1}

Evolution of thyroid radiological density and thyroid function in a patient with amiodarone induced thyrotoxicosis. In all CT scans radiologic density was measured in the same selected area in the right thyroid lobe. Density of the vertebral bone is used as a visual internal control, to demonstrate that the increase of thyroid density is not artefactual. Measurements of TSH, fT4 and fT3 show the combined evolution with time of thyroid density, size and function. The patient was first exposed to 3 months of amiodarone treatment, which was stopped for 3 months and then reinitiated for 17 months. The $x$ axis is numbered from the start of the first amiodarone treatment. The evolution of thyroid radiologic density during the first 6 months of amiodarone treatment is not shown, but the density of the thyroid before initiation of the first treatment is shown in the upper left part of the figure.

the first analysis of the evolution of intrathyroid iodine accumulation throughout the time course of AIT, showing a close association between thyroid radiologic density, size and function, with radiologic density and also volume, both rising before detection of thyrotoxicosis and then decreasing as a normal thyroid function was restored (Fig. 1).

The clinical story of the patient is in favor of AIT type 2 (thyroiditis) rather than AIT type 1 (increase in thyroid hormone synthesis), as the time between the first introduction of amiodarone and detection of thyrotoxicosis was 23 months (a first 3-month treatment was stopped for 3 months and then resumed for 17 months) and as the patient had no thyroid abnormality prior to amiodarone treatment (2). However, as thyrotoxicosis did not respond quickly to $0.5 \mathrm{mg} / \mathrm{kg} /$ day prednisone, an antithyroid treatment was added, as proposed by the European Thyroid Association (ETA) guidelines (2).

Van den Bruel et al. proposed that the high thyroid radiologic density in AIT might be due to lysosome trapping of amiodarone (1). One could also propose that it might rather be related to uptake by the thyroid of the large excess of iodine released by amiodarone in the circulation. According to this hypothesis, AIT type 2 could be viewed as a disease linked to an extremely high intrathyroid iodine accumulation, with intrafollicular accumulation of iodinated thyroglobulin, T4 and T3, which are finally released in the circulation when the 'burst' of thyroiditis occurs.

In conclusion, we show that the evolution of unenhanced radiological thyroid gland density is closely related to the evolution of thyroid function throughout the whole time course of AIT. This suggests that measurement of thyroid gland radiological density might predict the development and remission of AIT.

\section{Declaration of interest}

The authors declare that there is no conflict of interest that could be perceived as prejudicing the impartiality of this case report.
This work is licensed under a Creative Commons Attribution-NonCommercial 4.0 International dejcensem Bioscientifica.com at 04/26/2023 11:05:42AM 
Funding

This work did not receive any specific grant from any funding agency in the public, commercial or not-for-profit sector.

\section{Consent}

Written consent has been obtained from the patient for publication of the results of procedures which had been used for his care.

\section{Ethical appwroval}

Study ethics approval was obtained on August 18, 2021 (CECIC RhôneAlpes-Auvergne, Clermont-Ferrand, IRB 5891).

\section{References}

1 Van den Bruel A, Delanote J, Braeckman A, De Vroe C, Pyfferoen L, Ghekiere J, Duytschaever M \& Tavernier R. 'White thyroid' on unenhanced computed tomography in amiodarone-induced thyrotoxicosis type 2. Thyroid 201828 769-772. (https://doi. org/10.1089/thy.2017.0651)

2 Bartalena L, Bogazzi F, Chiovato L, Hubalewska-Dydejczyk A, Links TP \& Vanderpump M. 2018 European Thyroid Association (ETA) guidelines for the management of amiodarone-associated thyroid dysfunction. European Thyroid Journal 20187 55-66. (https://doi. org/10.1159/000486957)

Received in final form 30 September 2021

Accepted 12 October 2021

Accepted Manuscript published online 13 October 2021
This work is licensed under a Creative Commons Attribution-NonCommercial 4.0 International

dedcense Bioscientifica.com at 04/26/2023 11:05:42AM 